\title{
Relationship between Coloration of Polypropylene Gels and Wavelength Dispersions of Refractive Indices of Components
}

\author{
Hitoshi FuJIMATSU, ${ }^{\dagger}$ Yukinori IDETa, Hitoshi NAKAMURA, Hisanao USAMI, and \\ Shinji OGASAWARA \\ Department of Fine Materials Engineering, Faculty of Textile Science and Technology, \\ Shinshu University, 3-15-1 Tokida, Ueda, Nagano 386-8567, Japan
}

(Received July 17, 2000; Accepted September 19, 2000)

\begin{abstract}
The coloration of polypropylene gel was investigated by light scattering theory, in which wavelength dispersion of refractive indices for polypropylene and solvent is particularly noted. The coloration and thermochromic phenomena of polypropylene gel were concluded to be caused by the selective light scattering from size-controlled polypropylene networks with a solvent, that have the same refractive index as that of polypropylene at a certain wavelength in the visible region, but with degree of wavelength dispersion larger than that of polypropylene, since maximum transmittance wavelength in transmittance spectra of the gels obtained experimentally is in fair agreement with that estimated from wavelength dispersion curves of refractive indices of solvent and polypropylene obtained from independent experiments. The change of the transmittance spectra at a definite temperature with the elapse of time reflects change of the refractive index in the vicinity of the interface of polypropylene network in the gel prepared under confinement and change of the interface structure of the polypropylene networks in the gel.

KEY WORDS Coloration / Thermochromism / Polypropylene / Xylene / Wavelength Dispersion of Refractive Index
\end{abstract}

We have previously reported that polymer gels in organic solvents such as polypropylene ${ }^{1}$ and poly-1butene $^{2}$ develop color, although polypropylene and poly1-butene themselves are colorless, which changes with increase in temperature, where coloring is considered due to selective light scattering, as transmitted-light spectra of the gels approximately forms a reflected image of the scattered-light spectra, measured in the direction perpendicular to incident light. A similar phenomenon was found by Osada et al. ${ }^{3}$ for gels of copolymers of acrylic acid and methyl acrylate. The coloration mechanism has not been defined to. Tsujii et al. ${ }^{4,5}$ reported hydrophobic surface active agents develop iridescent coloration in dilute aqueous solutions. The coloration mechanism is explained on the basis of the interference of reflected light from the bilayer membranes of the molecules. When the white light is irradiated, color from solution changes according to the observing direction. The mechanism is inapplicable to the coloration of polyolefin gels because color from the gels is independent of direction.

Hayashi et al. ${ }^{6}$ recently reported the coloration of porous poly(vinyl alcohol)-poly(vinyl acetate) composites in organic solvents, and concluded that coloration is due to scattered light based on differences between refractive indices of polymer and solvent.

In the present work, the coloration of the polypropylene gel was investigated by light scattering theory, in which wavelength dispersion of refractive indices for polypropylene and solvent is particularly noted.

\section{EXPERIMENTAL}

\section{Materials \\ Polypropylene in this study was narrower dispersed}

${ }^{\dagger}$ To whom correspondence should be addressed. isotactic polypropylene with the weight average molecular weight of $20.0 \times 10^{4}$, and supplied by Research and Institute Center in Chisso Petrochemical Co. Organic solvents such as $o$-xylene, $o$-dichlorobenzene and tetrachloromethane were used to examine the coloration of polypropylene gel.

Polypropylene gel was prepared in a glass tube as follows: $0.2 \mathrm{~g}$ polypropylene and $10 \mathrm{~mL}$ solvent were placed in the glass tube, which was sealed by flame. After polypropylene was dissolved completely at $160^{\circ} \mathrm{C}$, polypropylene solution was quenched rapidly to liquid nitrogen temperature.

\section{Methods}

Wavelength dependent transparency was measured using a Shimadzu MPS-2000 spectrophotometer. Temperature was controlled by circulating water through the cell jacket. The light scattered spectrum of the gel in a direction perpendicular to incident light was measured using a Shimadzu RF-5000 fluorescent spectrophotometer with a temperature controller. The spectra were incorporated into the memory of a computer to be corrected by the spectrum of Xenon-lamp, the light source of the spectrophotometer.

The refractive indices of organic solvents were measured at wavelengths of 420 to $680 \mathrm{~nm}$ at constant room temperature of $20^{\circ} \mathrm{C}$ in experiments on the coloration of polypropylene gels in mixed solvents at $10^{\circ} \mathrm{C}$ to $50^{\circ} \mathrm{C}$ in experiments on thermochromic phenomenon of polypropylene gels, using an Atago Abbe-type refractometor Type-1 T equipped with a spectroscopic monochrometer.

Electron micrographs of gels were obtained with an Akashi type ALPHA 30 A scanning electron microscope. The specimens were prepared by depositing gold under a vacuum below $10^{-5}$ Torr after polypropylene gels were freeze-dried. 


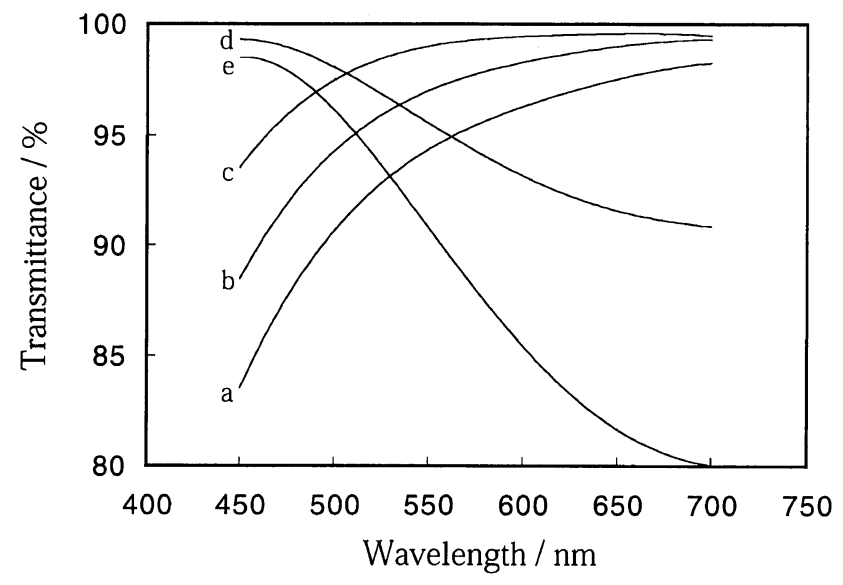

Figure 1. Transmittance spectra for $2 \mathrm{~g} / \mathrm{v}-\%$ polypropylene gel in $o$-xylene after preparing by cooling at $-195^{\circ} \mathrm{C}$, and reached equilibrium at different temperatures: (a) $10^{\circ} \mathrm{C}$, (b) $20^{\circ} \mathrm{C}$, (c) $30^{\circ} \mathrm{C}$, (d) 40 ${ }^{\circ} \mathrm{C}$, (e) $50^{\circ} \mathrm{C}$.

\section{RESULTS AND DISCUSSION}

\section{Thermochromism}

Polypropylene gels were prepared by quenching rapidly to $-195^{\circ} \mathrm{C}$ after the polypropylene was dissolved completely at $160^{\circ} \mathrm{C}$. The gels were used for the experiments after reaching equilibrium. Figure 1 shows transmittance spectra of $2 \mathrm{~g} / \mathrm{v}-\%$ polypropylene gels in $o$ xylene after reaching equilibrium at various temperatures. The spectrum with larger transparency on the long wavelength side in lower temperature changed gradually to that having larger transparency on the short wavelength side with increase in temperature. Light scattered spectra shown in Figure 2 approximately formed a reflected image of the spectra in Figure 1. This indicates that coloring phenomenon of the gels is due to selective scattering, but not to optical absorption, as reported previously. ${ }^{1,2}$

Change in the shape of light scattered spectrum with temperature corresponds fairly to change in color observed visually. Colors of the transmitted light and scattered light when white light was irradiated from back of gels are listed in Table I. Colors of transmitted and scattered light at the same temperature showed a complementary relationship to each other.

When coloration is due to light scattering, structure favorable to scattering visible light in the gel must be present. Thus SEM observation of the freeze-dried gel was carried out. In Figure 3, SEM images of the gel observed are shown. From the microphotographs, the gel is composed of expanded star-like crystals with a diameter of submicron order. Polypropylene networks of this order have structures favorable to scattering of visible light.

\section{Thermo-Reversibility}

As the coloration of the polypropylene gel develops through the microstructure in the gel prepared under the conditions above, there may be a limit to thermal reversibility of the gel. Figure 4 shows a DSC thermogram for $2 \mathrm{~g} / \mathrm{v}-\%$ of polypropylene gel in $o$-xylene. Endothermic deviation corresponding to fusion of polypropylene crystallite in the gel begins to occur near $70^{\circ} \mathrm{C}$, and

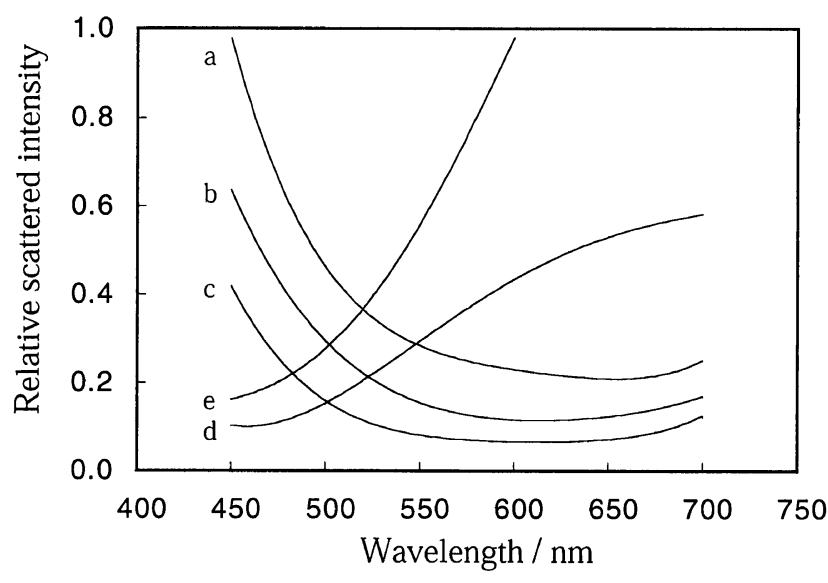

Figure 2. Light scattered spectra for $2 \mathrm{~g} / \mathrm{v}-\%$ polypropylene gel in $o$-xylene after preparing by cooling at $-195^{\circ} \mathrm{C}$, and reached equilibrium at different temperatures: (a) $10^{\circ} \mathrm{C}$, (b) $20^{\circ} \mathrm{C}$, (c) $30^{\circ} \mathrm{C}$, (d) $40^{\circ} \mathrm{C}$, (e) $50^{\circ} \mathrm{C}$.

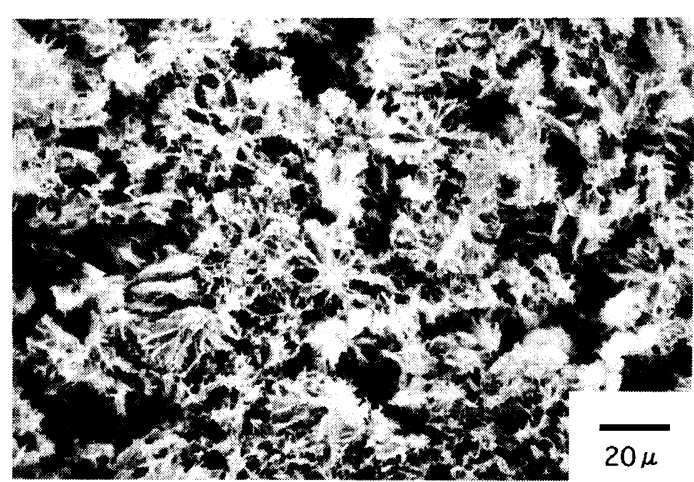

(a)

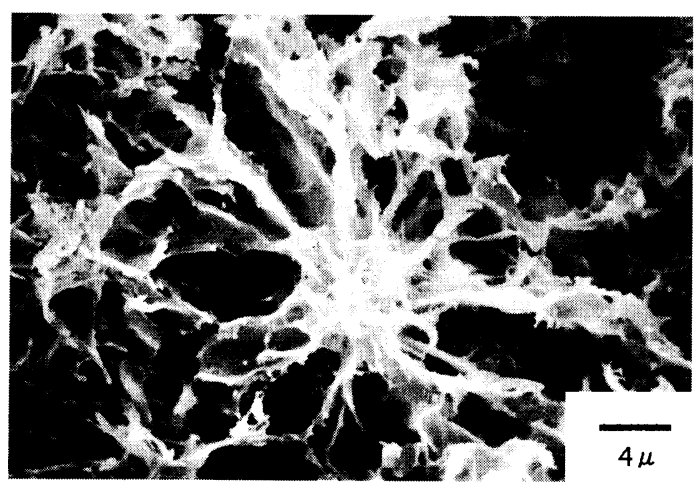

(b)

Figure 3. SEM images for $2 \mathrm{~g} / \mathrm{v}$ - $\%$ polypropylene gel in $o$-xylene prepared by quenching at $-195^{\circ} \mathrm{C}$. Magnification: (a) $\times 500$, (b) $\times$ 2500 .

Table I. Colors of transmitted and scattered light of $2 \mathrm{~g} / \mathrm{v}-\%$ polypropylene gel in $o$-xylene after reached equilibrium at various temperatures, which prepared by cooling at $-195^{\circ} \mathrm{C}$

\begin{tabular}{cll}
\hline \multirow{2}{*}{$\begin{array}{c}\text { Temperature } \\
/{ }^{\circ} \mathrm{C}\end{array}$} & \multicolumn{2}{c}{ Color } \\
\cline { 2 - 3 } & Transmitted light & Scattered light \\
\hline 10 & Deep yellow & Blue \\
20 & Golden yellow & Blue violet \\
30 & Light green & Red violet \\
40 & Blue & Yellow \\
50 & Whitish clouding & Whitish clouding \\
\hline
\end{tabular}




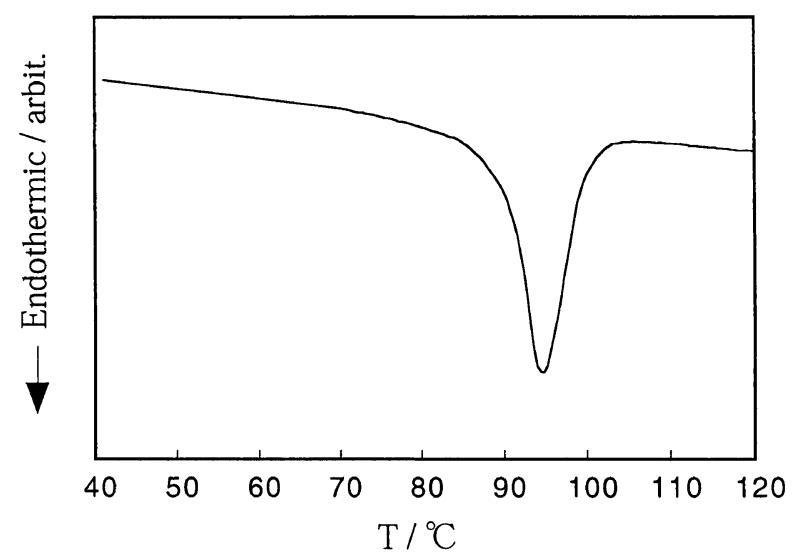

Figure 4. DSC thermogram for $2 \mathrm{~g} / \mathrm{v}-\%$ polypropylene gel in $o$ xylene after preparing by cooling at $-195^{\circ} \mathrm{C}$, and reached equilibrium at $30^{\circ} \mathrm{C}$.

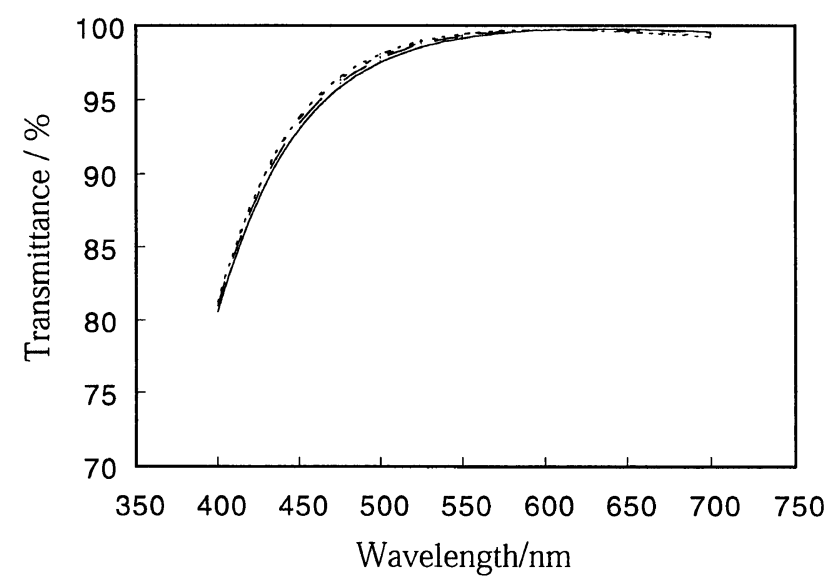

Figure 5. Time dependency of transmittance spectrum for $2 \mathrm{~g} / \mathrm{v}-$ $\%$ of polypropylene gel in $o$-xylene prepared by cooling at $-195^{\circ} \mathrm{C}$ and maintained at $30^{\circ} \mathrm{C}$ for $2 \mathrm{~h}(-), 4 \mathrm{~h}(--), 6 \mathrm{~h}(\cdots), 10 \mathrm{~h}$ $(\cdots \cdots)$.

peaks at $94.4^{\circ} \mathrm{C}$.

Heating beyond $70^{\circ} \mathrm{C}$ is thus presumed to induce reduction or the disappearance of coloration. Figures 5 and 6 show time dependency of the transmittance spectra of $2 \mathrm{~g} / \mathrm{v}-\%$ polypropylene gels in $o$-xylene prepared by rapid cooling at $-50^{\circ} \mathrm{C}$, on maintained at $30^{\circ} \mathrm{C}$ and $40^{\circ} \mathrm{C}$, respectively. The spectrum for the gel kept at $20^{\circ} \mathrm{C}$ was independent of time and maintained the initial. In Figure 5 , the spectrum of the gel kept at $30^{\circ} \mathrm{C}$ changes slightly with time, reaching a steady state after $6 \mathrm{~h}$. For the gel kept at $40^{\circ} \mathrm{C}$, the shape of the spectrum is different from that at $30^{\circ} \mathrm{C}$, and the spectrum changes significantly with time and reaches a steady state at $16 \mathrm{~h}$. The spectrum after reaching a steady state was unchanged with time when temperature was lower than or the same as that for a steady state. Similar results were obtained up to $70^{\circ} \mathrm{C}$. This suggests that microstructure in the gel changes gradually with time at $30^{\circ} \mathrm{C}$ to $70^{\circ} \mathrm{C}$, and once it reaches a steady state, is unchanged unless heated beyond the temperature required to stabilize the gel.

\section{Effect of Solvent}

To examine how a solvent associates with coloration, polypropylene gels in a variety of solvents were pre-

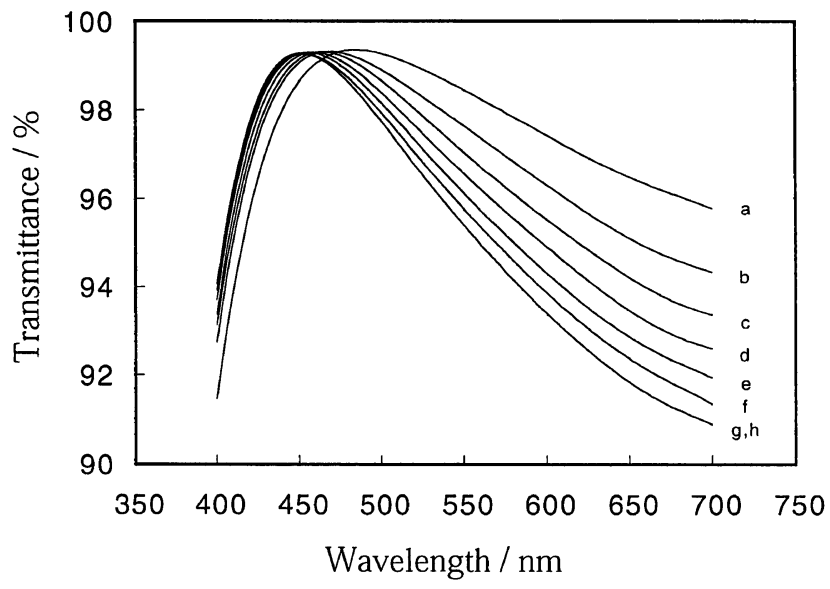

Figure 6. Time dependency of transmittance spectrum for $2 \mathrm{~g} / \mathrm{v}$ $\%$ polypropylene gel in $o$-xylene prepared by cooling at $-195^{\circ} \mathrm{C}$ and maintained at $40^{\circ} \mathrm{C}$ for (a) $2 \mathrm{~h}$, (b) $4 \mathrm{~h}$, (c) $6 \mathrm{~h}$, (d) $8 \mathrm{~h}$, (e) $10 \mathrm{~h}$, (f) $12 \mathrm{~h}$, (g) $14 \mathrm{~h}$, (h) $16 \mathrm{~h}$.

Table II. List of solvents developed coloring for polypropylene gel prepared by quick cooling

\begin{tabular}{lc}
\hline \multicolumn{1}{c}{ Solvent } & Coloring \\
\hline Benzene & ++ \\
Toluene & ++ \\
$o$-Xylene & ++ \\
$m$-Xylene & ++ \\
$p$-Xylene & ++ \\
Decalin & - \\
Tetralin & + \\
Cyclohexane & - \\
Tetrachloroethylene & ++ \\
$o$-Dichlorobenzene & - \\
1,1,2,2-Tetrachloroethane & - \\
Chlorobenzene & + \\
Carbontetrachloride & -- \\
\hline
\end{tabular}

++ : Strikingly coloring, $+:$ Weakly coloring,

- : Gelling but no coloring, - - : No gelling and no coloring.

pared. The results are shown in Table II. Solvents that develop coloration were limited to aromatic and chlorinated hydrocarbon compounds. Solvents, such as decalin, and $o$-dichlorobenzene, cannot develop coloration although they gel polypropylene. The colors of gels were found to differ, dependent on the solvent, even at the same temperature.

To examine how a solvent associates with the polypropylene networks in the gel, the displacement of a solvent in gel by other solvents is useful. If the microstructure in the gel formed is established by the solvent and temperature condition, after polypropylene gel was prepared, displacement of the solvent by other solvents reveals the effect of solvent on the microstructure. Abrupt displacement of a solvent in the gel by the other solvent is liable to induce change of the microstructure in the gel, and thus the solvent in the gel was cautiously displaced by mixed solvents while increasing solvent to be displaced. As example of polypropylene gel in benzene, when benzene in the gel was displaced by toluene, the color of the toluene-displaced gel was the same as that of polypropylene gel prepared using toluene. This suggests that coloration is induced by interaction between solvent and microstructure in the gel. 
Selective Light Scattering and Wavelength Dispersion of Refractive Indices of Solvent and Polypropylene

Rayleigh-Gans-Debye approximation ${ }^{7-10}$ on light scattering from colloidal particles was made by the following equations; when the refractive index of the particle approximately equal to that of medium, specific scattered intensity ${ }^{11}$ of spherical particles of any size:

$$
\begin{gathered}
r^{2} I / I_{0} C=3 / 2 P^{2} \lambda \alpha^{3} d_{2} \cdot i \cdot P(\gamma), \\
P=a / b, \alpha=2 \pi a / \lambda,
\end{gathered}
$$

where $I$ is intensity of the light scattered at angle $\gamma, I_{0}$ intensity of incident light, $C$ concentration of a solution, $a$ diameter in rotational axial direction of particle, $b$ the diameter in the direction normal to a particle, $\lambda$ wavelength of incident light, $d_{2}$ being the density of a solute, $i$ intensity of the light scattered from one particle, is given,

$$
\begin{gathered}
i=\left[\left(m^{2}-1\right) /\left(m^{2}+2\right)\right]^{2} \alpha^{6}\left[\left(1+\cos ^{2} \gamma\right) / 2\right], \\
m=\mu_{1} / \mu_{2},
\end{gathered}
$$

where $\mu_{1}$ is the refractive index of a solvent, $\mu_{2}$ is the refractive index of a solute, $P(\gamma)$ in eq 1 is an interference factor for taking into account interference induced by particles observed in the direction at an angle $\gamma . i$ in eq 2 depends the relative refractive index between solute and solvent. Therefore, no light scattering occurs when the refractive index of particles in colloidal suspension is the same as that of a solvent. If wavelength dispersion of the refractive index for a particle and solvent differs, light scattering will depend wavelength.

Coloration of polypropylene gel was investigated by wavelength dispersion of refractive indices of solvent and polypropylene using the light scattering theory. The refractive index of polypropylene $\mathrm{n}_{\mathrm{D}}^{20}$ is approximately 1.50 , and thus $o$-xylene and tetrachloromethane as solvents were used and refractive index higher and lower than that of polypropylene, respectively.

Gels of polypropylene were prepared using solvents mixed at varying ratios. Colors of the gels under irradiation of white light changed from blue to yellow through blue violet and red violet as tetrachloromethane content increased. The transmitted-light spectra are shown in Figure 7. Change of the spectra explains well that of color observed visually.

Wavelength dispersion of mixed solvents at various temperatures was measured by an Abbe refractometer equipped with a spectroscopic monochromator. The results are shown in Figure 8. Dispersion curves decreased as wavelength increased, and shifted to lower direction as tetrachloromethane content increased. Wavelength dispersion of the refractive index of polypropylene in the gel was estimated by maximum-transmittance, using maximum transmittance obtained when the refractive index of suspended particle was the same as that of solvent. Transmittance of polypropylene gels using $o$ xylene-tetrachloromethane mixtures with varying mixed ratios was measured at various wavelengths. Mixed ratios can be converted to refractive indices when refractive indices are measured. Refractive index dependence

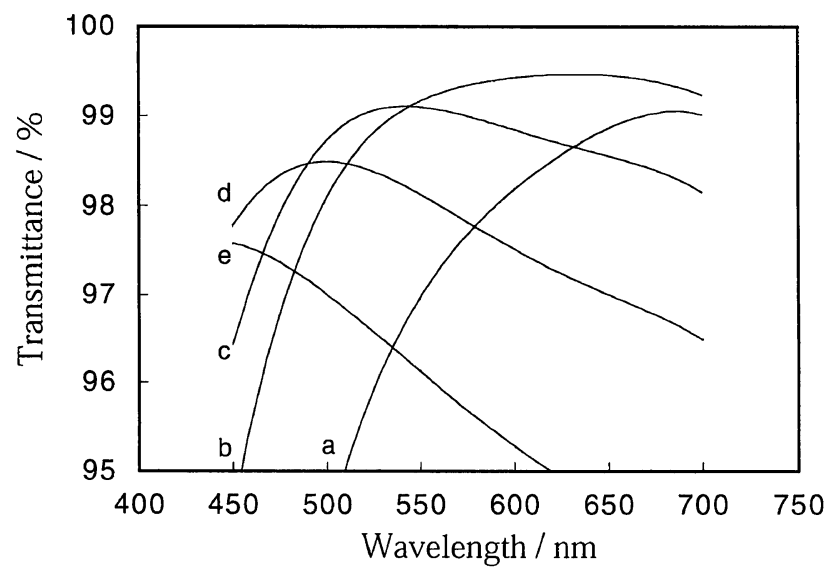

Figure 7. Transmittance spectra for $2 \mathrm{~g} / \mathrm{v}-\%$ of polypropylene gels in $o$-xylene-tetrachloromethane mixtures with different tetrachloromethane content after preparing by cooling at $-195^{\circ} \mathrm{C}$, and reached equilibrium at $20^{\circ} \mathrm{C}$, and: (a) $0 \%$, (b) $10 \%$, (c) $20 \%$, (d) $30 \%$, (e) $40 \%$.

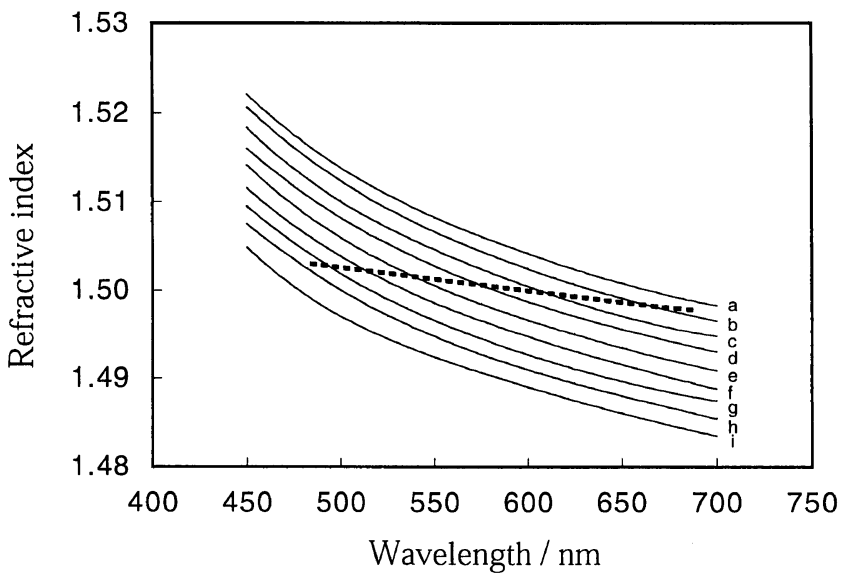

Figure 8. Wavelength dispersion of the refractive indices for both $o$-xylene-tetrachloromethane mixtures with different tetrachloromethane content (solid lines) and polypropylene (dotted line) obtained by maximum-transmittance: (a) $0 \%$, (b) $5 \%$, (c) $10 \%$, (d) $15 \%$, (e) $20 \%$, (f) $25 \%$, (g) $30 \%$, (h) $35 \%$, (i) $40 \%$.

of transmittance of polypropylene gel at $575 \mathrm{~nm}$ is shown in Figure 9. The refractive index of the solvent that gives maximum-transmittance corresponds to that of polypropylene in the gel. Wavelength dispersion of the refractive index of polypropylene obtained in this way is shown in Figure 8 as a dotted line. The slope of the dispersion curve for polypropylene is less than for solvents, and thus polypropylene curve crosses some curves of solvents. The cross point shifts to the shorter wavelength side with increase in tetrachloromethane. Cross points are in fair agreement with peak values of the spectra in Figure 7. These results support strongly the possibility that coloration develops by selective light scattering based on differences in dispersion curves of the refractive indices of polypropylene and solvents.

Relationship between Thermochromism and Wavelength Dispersion of Refractive Index

When coloring mechanism derived in preceding section is valid, thermochromic phenomenon of the gel must 


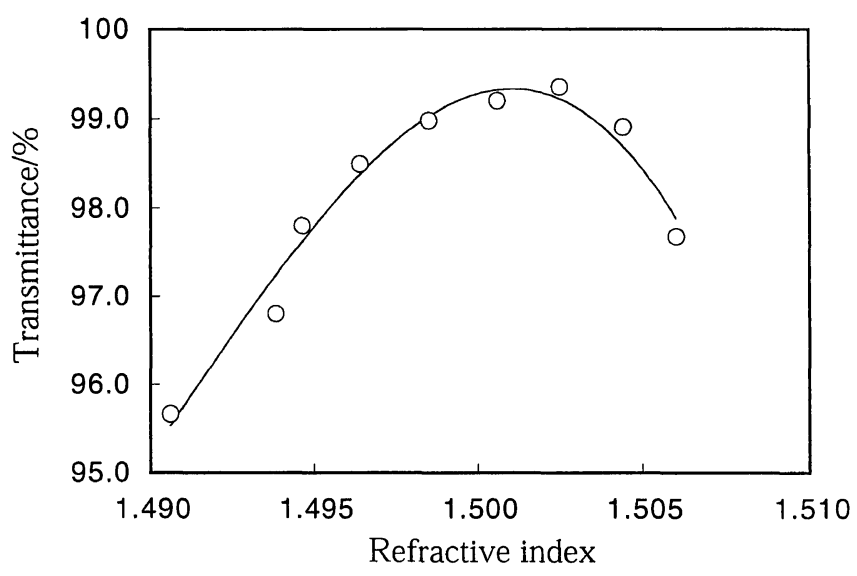

Figure 9. Plots of transmittance of polypropylene gels in $o$ xylene- tetrachloromethane mixed solvents against refractive indices of mixtures in light of $575 \mathrm{~nm}$.

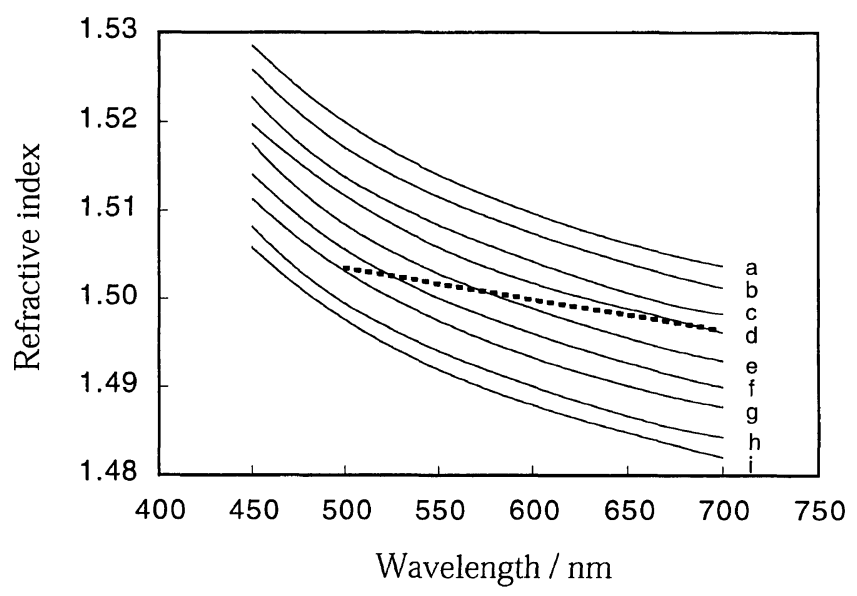

Figure 10. Wavelength dispersion of the refractive indices for both $o$-xylene (solid lines) at different temperatures and polypropylene (dotted line) in the gel obtained by maximumtransmittance: (a) $10^{\circ} \mathrm{C}$, (b) $15^{\circ} \mathrm{C}$, (c) $20^{\circ} \mathrm{C}$, (d) $25^{\circ} \mathrm{C}$, (e) $30^{\circ} \mathrm{C}$, (f) 35 ${ }^{\circ} \mathrm{C}$, (g) $40^{\circ} \mathrm{C}$, (h) $45^{\circ} \mathrm{C}$, (i) $50^{\circ} \mathrm{C}$.

be explained by the same logic. The validity of the coloring mechanism was investigated using polypropylene gel in $o$-xylene. Wavelength dispersion of the refractive index of $o$-xylene was measured at $10^{\circ} \mathrm{C}$ to $40^{\circ} \mathrm{C}$. The results are shown in Figure 10. The refractive index of $o-$ xylene was reduced as the wavelength increased. The dispersion curve shifted to lower direction with increase in temperature.

Refractive index of polypropylene in the gel in $o$ xylene was estimated by maximum-transmittance as follows. Transparency of the gels was measured at different temperatures and wavelengths. Transparencytemperature curve at definite wavelengths were converted to transparency-refractive indices of $o$-xylene curve. The refractive index of the peak of the curve is regarded as the same as that of polypropylene in the gel. The values at various wavelengths are shown by dotted lines in Figure 10. The slope of the dispersion curve for polypropylene is smaller than for solvents and the dispersion curve of polypropylene thus crosses some curves of the solvent at different temperatures. The cross point shifts to shorter wavelength side with increase in tem- perature. The color of the gel thus changes with temperature. The cross points approximately agree with peak values of transmittance spectra of the polypropylene gel in $o$-xylene measured at corresponding temperatures, as shown in Figure 1. Because the gels kept at different temperatures were used for determining maximum transmittance. That is, change of the refractive index of polypropylene in the gel on kept at different temperatures is not considered. The dispersion curve of the refractive index at each temperature must be measured. The result proves that the thermochromic phenomenon of polypropylene gel may be explained by selective light scattering from the polypropylene networks in the gel.

\section{CONCLUDING REMARKS}

Maximum transmittance wavelengths in transmittance spectra of the gel obtained experimentally were in fair agreement with those for wavelength dispersion curves of refractive indices of the solvent and polypropylene. Thus, coloration and thermochromic phenomena of the polypropylene gel were found due to selective light scattering from size-controlled polypropylene networks with solvent, having the same refractive index as that of polypropylene at a certain wavelength in the visible region. Change of the transmittance spectra at a definite temperature with time is considered to reflect change of the refractive index near the interface of polypropylene network in the gel prepared under confinement. After the transmittance spectra reached a steady state at $20^{\circ} \mathrm{C}$ to $70^{\circ} \mathrm{C}$, were reversible unless heated beyond the temperature required to stabilize the gel. They suggest that once the interface structure of the polypropylene networks in the gel reaches a steady state, it is unchanging unless heated beyond the temperature required to stabilize the gel.

The refractive index of materials varies sensitively with temperature, because the density of a dielectric varies with temperature. Variation in refractive index with temperature for solid and liquid reflects that variation in density with temperature ${ }^{12}$ is of the order of $10^{-4}$ and $10^{-3}$. Variation in aromatic compounds is larger than for aliphatic compounds. Larger variation is useful for development of clear colors at limited temperature.

Acknowledgment. This work was supported in part by Grant-in-Aid for COE Research (10CE2003) by the Ministry of Education, Science, Sports, and Culture of Japan.

\section{REFERENCES}

1. H. Fujimatsu and S. Kuroiwa, Colloid Polym. Sci., 265, 938 (1987).

2. H. Fujimatsu, S. Ogasawara, H. Ihara, T. Takashima, K. Toyaba, and S. Kuroiwa, Colloid Polym. Sci., 266, 688 (1988).

3. H. Okazaki and Y. Osada, Prepr. in the 61st Conf. Jpn. Chem. Soc., 1, 304 (1991).

4. N. Satoh and K. Tsujii, J. Phys. Chem., 91, 6629 (1987).

5. N. Satoh and K. Tsujii, Langmuir, 8, 581 (1992).

6. S. Hayashi, J. Xu, K. Asada, and T. Hirai, J. Colloid Interface Sci., 163, 315 (1994).

7. L. Rayleight, Proc. Roy. Soc., A84, 25 (1911). 


\section{H. Fujimatsu et al.}

8. R. Gans, Ann. Phys., 76, 29 (1925).

9. P. Debye, Menke, and Forts. Roentgenforschung, 2, 1 (1931).

10. P. Debye, J. Phys. Colloid. Chem., 51, 18 (1947).
11. Vande Hulst, "Light Scattering by Small Particles", John Wiley and Sons, Inc., New York, N.Y., 1957, pp 85-100. 12. W. Heller, J. Chem. Phys., 14, 9 (1946). 